\title{
Does a lung cancer screening programme promote smoking cessation?
}

\author{
Filippo Lococo, ${ }^{1}$ Giuseppe Cardillo, ${ }^{2}$ Giulia Veronesi ${ }^{3}$
}

Lung cancer is a preventable form of cancer with approximately $90 \%$ of cases attributable to cumulative tobacco exposure. ${ }^{1}$ Smoking cessation and early detection of lung cancer remain critical goals for lung cancer prevention and control. Indeed, the health benefits of smoking cessation reach well beyond reducing the risk of lung cancer, ${ }^{2}$ resulting in an overall reduction of tobacco-related mortality. Unfortunately, smoking cessation delivery and patient adherence is still a major challenge.

While there is a body of evidence supporting smoking cessation (primary prevention) and early detection of lung cancer (secondary prevention) to decrease lung cancer mortality, data on the effectiveness and interaction between smoking cessation and lung cancer screening are still sparse and inconsistent. ${ }^{3-6}$ The administration of a rigorous smoking cessation intervention (based on the combination of counselling and drug treatment) to a lung cancer screening population may lead to high smoking cessation rates (up to $30 \%{ }^{3}$ ).

Published in Thorax, the UK Lung Cancer Screening (UKLS) trial group report the effect of low-dose CT screening on smoking cessation. ${ }^{7}$ The UKLS trial assessed the feasibility, cost-effectiveness and psychosocial impact of lung cancer screening using a single low-dose CT screen versus no screening in a UK highrisk population. The authors have investigated the behavioural effect of trial participation on smoking cessation in both the short term and long term.

The initial sample consisted of 247354 individuals, aged 50-75 years, from six recruitment sites in the UK. The subjects received trial information packs, including a self-report questionnaire regarding lung

\footnotetext{
${ }^{1}$ Unit of Thoracic Surgery, Arcispedale Santa Maria Nuova-IRCCS, Reggio Emilia, Italy

${ }^{2}$ Unit of Thoracic Surgery, Azienda Ospedaliera S. Camillo Forlanini, Lazzaro Spallanzani Hospital, Rome, Italy

${ }^{3}$ Division of Thoracic Surgery, European Institute of Oncology, Milan, Italy

Correspondence to Dr Filippo Lococo, Unit of Thoracic Surgery, Arcispedale Santa Maria NuovaIRCCS, via Risorgimento 80, 42100 Reggio Emilia, Italy; filippo_lococo@yahoo.it
}

cancer risk factors. From the questionnaire responders, 8729 individuals were identified as 'high-risk' subjects for lung cancer using a mathematical prediction model. Then, high-risk subjects were invited to attend their local recruitment centre and those who gave informed written consent were randomised on a 1:1 ratio to the screening intervention or control non-screening arms. Of a total of 4055 subjects randomised to CT screening or control, 1546 were baseline smokers (759 intervention and 787 control). Of major clinical relevance, the authors observed that 2 weeks after receiving either a letter of assignment to the control group or a baseline CT scan result letter (T1), the short-term smoking cessation rate was higher in the intervention screening arm when compared with the control group (10\% vs 5\%; adjusted OR (aOR) 2.38, $\mathrm{p}<0.001)$.

On the one hand, the subjects assigned to the non-screening control group exhibited limited change in their behavioural tobacco use (only 5\% quit smoking) despite standard smoking cessation advice leaflets and a list of local NHS Stop Smoking services offered to them. The smoking cessation rate in these subjects was similar to the population not involved in a lung cancer screening programme. ${ }^{8}$ Smoking cessation, greater than 6 months, was observed in $4 \%-7 \%$ of smokers without a specific intervention programme. ${ }^{8}$ On the other hand, in the subjects assigned to the intervention screening arm, it resulted in a higher smoking cessation rate. Enrolment in the screening programme elicited a response in the subjects with the effect of quitting smoking in $10 \%$ at the time of receiving the letter detailing the CT scan result and $15 \%$ at the end of the study. In addition to these important short-term clinical observations, the UKLS screening trial data demonstrated longerterm smoking cessation rates in the intervention group; within those subjects who required further investigation after their initial CT, those with an abnormal scan were more likely to quit smoking when compared with the control group (aOR 2.29, $\mathrm{p}=0.007)$ and those receiving a normal scan result (aOR 2.57, $\mathrm{p}<0.001)$.
Despite these current data, ${ }^{7}$ the interaction between smoking cessation and lung cancer screening is still a controversial area. ${ }^{49}$ Previous data ${ }^{10}$ have suggested that taking part in lung screening may provide reassurance to the subject and providing a 'licence to smoke', in particular, for smokers who receive favourable screening results. Indeed, one possible drawback of lung cancer screening may be that it can induce a false sense of safety and security in the participants so that they may feel protected against the harmful effect of smoking. ${ }^{11}$ Thus, a normal CT scan result may reduce the motivation of a smoker to quit and inadvertently encourage continued smoking. However, this concern has never been confirmed in the lung cancer screening programmes.

A teachable moment in healthcare is defined as a health event that motivates an individual to adopt a risk-reducing health behaviour. Enrolment in a screening programme offers the opportunity to inform the participants of the harmful effects of smoking and from this increase their motivation to quit smoking. ${ }^{10} 12 \mathrm{In}$ the analysis of UKLS trial results, it clearly emerges that enrolling in a lung cancer screening programme has an overall positive effect on smoking cessation, especially in subjects with an abnormal CT result when additional clinical investigations are needed.

In detail, the authors observed a statistically significant effect of additional clinical investigation on long-term (T2) smoking cessation in univariable $(\mathrm{p}<0.001)$ and multivariable $(p<0.001)$ analysis. Such subjects have their awareness as 'high-risk' individuals increased, which represents an important teachable moment. To simplify this, the scare of an abnormal CT scan with the fear of a potential lung cancer diagnosis, and education from a physician about lung cancer with the dangers of continued smoking provide the motivation to reduce smoking habits in these subjects.

The Dutch-Belgian screening study $(\mathrm{NELSON})^{13}$ and the Danish Lung Cancer Screening Trial $^{14}$ have also investigated the effect of lung cancer screening on smoking habits. Neither trial demonstrated a difference in smoking cessation between the screening group and the control group. However, there is some evidence that a positive screening test result increases the smoking cessation rate. ${ }^{11}$ Our own data from the Cosmos-II trial (CT lung cancer screening in Italian population) support this. ${ }^{9}$ Similar to UKLS, we found that 'high-risk' subjects, according to a personalised risk model, 
requiring an additional CT scan were more likely to quit smoking compared with 'low-risk' subjects. Furthermore, the MILD trial ${ }^{15}$ demonstrated that the smoking cessation achieved during a lung cancer screening programme resulted in a reduction in mortality.

In conclusion, lung cancer CT screening is a teachable moment that serves as a strong catalyst for smoking cessation. A lung cancer screening programme including personalised smoking cessation interventions may therefore be the most effective method to reduce mortality from tobacco-related lung cancer and should be a public health priority.

Contributors FL conceived the ideas and wrote the editorial. GC and GV worked together to analysed and edited the paper.

Competing interests None declared.

Provenance and peer review Commissioned; internally peer reviewed.

(C) Article author(s) (or their employer(s) unless otherwise stated in the text of the article) 2017. All rights reserved. No commercial use is permitted unless otherwise expressly granted.

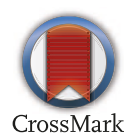

To cite Lococo F, Cardillo G, Veronesi G. Thorax 2017:72:870-871.

Published Online First 26 July 2017

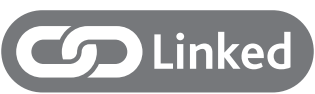

- http://dx.doi.org/10.1136/thoraxjnl-2016-209690

Thorax 2017:72:870-871

doi:10.1136/thoraxjnl-2017-210621

\section{REFERENCES}

1 Centers for Disease Control and Prevention. Lung cancer: risk factors. http://www.cdc.gov/cancer/lung/ basic_info/risk_factors.htm (accessed Jul 2013).

2 US Department of Health and Human Services, Centers for Disease Control and Prevention and Health Promotion. Office on Smoking and Health. The health consequences of smoking - 50 years of progress: a report of the Surgeon General. Washington, DC: USDHSS, 2014.

3 Deppen SA, Grogan EL, Aldrich MC, et al. Lung cancer screening and smoking cessation: a teachable moment? J Natl Cancer Inst 2014;106:dju122.

4 Ferketich AK, Otterson GA, King M, et al. A pilot test of a combined tobacco dependence treatment and lung cancer screening program. Lung Cancer 2012;76:211-5.

5 Ostroff JS, Buckshee N, Mancuso CA, et al. Smoking cessation following CT screening for early detection of lung cancer. Prev Med 2001;33:613-21.

6 Clark MM, Cox LS, Jett JR, et al. Effectiveness of smoking cessation self-help materials in a lung cancer screening population. Lung Cancer 2004;44:13-21.
7 Brain K, Carter B, Lifford KJ, et al. Impact of low-dose CT screening on smoking cessation among high-risk participants in the UK Lung Cancer Screening Trial. Thorax 2017:72:912-18.

8 US Department of Health and Human Services (2008). Treating tobacco use and dependence: 2008 update. Rockville: USDHHS, 2008.

9 Filippo L, Principe R, Cesario A, et al. Smoking cessation intervention within the framework of a lung cancer screening program: preliminary results and clinical perspectives from the "Cosmos-II" Trial. Lung 2015;193:147-9.

10 van der Aalst CM, van den Bergh KA, Willemsen MC, et al. Lung cancer screening and smoking abstinence: 2 year follow-up data from the Dutch-Belgian randomised controlled lung cancer screening trial. Thorax 2010;65:600-5.

11 Pedersen JH, Tønnesen P, Ashraf H. Smoking cessation and lung cancer screening. Ann Trans/ Med 2016;4:157.

12 Bach PB, Mirkin JN, Oliver TK, et al. Benefits and harms of $\mathrm{CT}$ screening for lung cancer: a systematic review. JAMA 2012;307:2418-29.

13 van der Aalst CM, van Klaveren RJ, van den Bergh $\mathrm{KA}$, et al. The impact of a lung cancer computed tomography screening result on smoking abstinence. Eur Respir J 2011;37:1466-73.

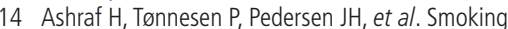
habits were unaffected by $\mathrm{CT}$ screening at 1-year follow-up in the Danish Lung Cancer Screening Trial (DLCST). Thorax 2009;64:371-2.

15 Pastorino U, Boffi R, Marchianò A, et al. Stopping smoking reduces mortality in low-dose computed tomography screening participants. J Thorac Oncol 2016;11:693-9. 